\title{
Local Stability Conditions for the Babuška Method of Lagrange Multipliers*
}

\author{
By Juhani Pitkäranta
}

\begin{abstract}
We consider the so-called Babuška method of finite elements with Lagrange multipliers for numerically solving the problem $\Delta u=f$ in $\Omega, u=g$ on $\partial \Omega, \Omega \subset R^{n}$, $n \geqslant 2$. We state a number of local conditions from which we prove the uniform stability of the Lagrange multiplier method in terms of a weighted, mesh-dependent norm. The stability conditions given weaken the conditions known so far and allow mesh refinements on the boundary. As an application, we introduce a class of finite element schemes, for which the stability conditions are satisfied, and we show that the convergence rate of these schemes is of optimal order.
\end{abstract}

1. Introduction. Let $\Omega$ be a bounded simply connected domain in $R^{n}, n \geqslant 2$, with smooth boundary $\partial \Omega$. We consider the second-order Dirichlet problem

$$
\Delta u=f \quad \text { in } \Omega, \quad u=g \text { on } \partial \Omega .
$$

If $f \in L_{2}(\Omega)$ and $g \in H^{r}(\partial \Omega), r>1$, the problem (1.1) admits the following variational formulation: find a pair $(u, \psi), u \in H^{1}(\Omega), \psi \in L_{2}(\partial \Omega)$, such that

$$
\begin{array}{r}
\int_{\Omega} \nabla u \cdot \nabla v d x-\int_{\partial \Omega} v \psi d s+\int_{\partial \Omega} u \varphi d s=-\int_{\Omega} f v d x+\int_{\partial \Omega} g \varphi d s, \\
\text { for all }(v, \varphi) \in H^{1}(\Omega) \times L_{2}(\partial \Omega) .
\end{array}
$$

If $(u, \psi)$ is the solution of problem (1.2), then $u$ is the weak solution of problem (1.1) and $\psi$ equals $\partial u / \partial n$, the normal derivative of $u$ on $\partial \Omega$ (cf. [1]).

If $M_{1}^{h} \subset H^{1}(\Omega)$ and $M_{2}^{h} \subset L_{2}(\partial \Omega)$ are finite-dimensional, the Lagrange multiplier method [1] , [2] for the approximate solution of problem (1.1) consists of seeking for a pair $\left(u_{h}, \psi_{h}\right) \in M_{1}^{h} \times M_{2}^{h}$ such that

$$
\begin{aligned}
\int_{\Omega} \nabla u_{h} \cdot \nabla v d x-\int_{\partial \Omega} v \psi_{h} d s+\int_{\partial \Omega} u_{h} \varphi d s=- & \int_{\Omega} f v d x+\int_{\partial \Omega} g \varphi d s \\
& \text { for all }(v, \varphi) \in M_{1}^{h} \times M_{2}^{h} .
\end{aligned}
$$

It is well known, cf. [1], [2] , [4], that the uniform stability of the Lagrange multiplier method can be achieved only by rather careful choices of the subspaces $M_{1}^{h}$ and $M_{2}^{h}$. In particular, if $M_{1}^{h}$ is fixed, then the choice of $M_{2}^{h}$ is critical [2]. In [1] it is shown that for finite element subspaces the stability can be achieved provided, essentially, that the diameter of the smallest element on $\partial \Omega$ and that of the largest element in the

Received January 31, 1979; revised March 7, 1980.

1980 Mathematics Subject Classification. Primary 65N30.

* This work was supported by the Finnish National Research Council for Technical Sciences. 
interior of $\Omega$ are related as

$$
d_{\min }(\partial \Omega) \geqslant C d_{\max }(\Omega)
$$

where $C$ depends on $\Omega$.

The problem of weakening the condition (1.4) was studied in [8], where it was shown that for quasiuniform finite element meshes in $R^{2}$ the constant $C$ in (1.4) can be allowed a value close to unity, with certain natural choices of the boundary subspaces. In the present paper we consider the more general situation where mesh refinements are allowed. Neither (1.4) nor the conditions given in [8] allow mesh refinements on $\partial \Omega$.

The technique we use is somewhat different from that of [1] or [8]. Instead of working with the Sobolev space $H^{-1 / 2}(\partial \Omega)$, we carry out the analysis using $L_{2}(\partial \Omega)$, supplied with certain weighted, mesh-dependent norms.

In Section 2 a number of technical assumptions are made, which are sufficient for the uniform stability of the Lagrange multiplier method. The assumptions are given in a form allowing them to be checked locally for subspaces of finite element type.

In Section 3 the uniform stability of the Lagrange multiplier method is proved from the assumptions of Section 2. Here the key result, which allows local conditions in the case of mesh refinements, is Lemma 3.2. The uniform stability yields immediately an abstract quasioptimal error bound for the approximate solution. As a by-product we obtain here a weighted convergence result for $u-u_{h}$ on the boundary.

The verification of the stability conditions in practice is finally considered in Section 4, where compatible finite element-type subspaces are constructed assuming a simplicial mesh in the interior of $\Omega$. Quasioptimal convergence rates are proved for the resulting finite element schemes.

Among other cases, where the conditions of Section 2 can be verified, we mention the boundary subspaces constructed in [8] and [9] for plane domains.

2. The Basic Assumptions. For a domain $\Omega \subset R^{n}$ we let $H^{k}(\Omega), k \geqslant 0, k$ integer, denote the usual Sobolev space with the norm

where

$$
\|u\|_{H^{k}(\Omega)}^{2}=\sum_{j=0}^{k}|u|_{j, \Omega}^{2},
$$

$$
|u|_{j, \Omega}^{2}=\sum_{|\alpha|=j} \int_{\Omega}\left(D^{\alpha} u\right)^{2} d x, \quad \alpha=\left(\alpha_{1}, \ldots, \alpha_{n}\right),|\alpha|=\alpha_{1}+\cdots+\alpha_{n} .
$$

For nonintegral $k$, the norm $\|\cdot\|_{H^{k}(\Omega)}$ is defined by interpolation [7].

For a definition of the spaces $H^{k}(\partial \Omega)$, cf. [1] . To define a convenient norm for $H^{k}(\partial \Omega)$, when $k$ is an integer, let $\left\{S_{v}\right\}$ be a finite family of open, disjoint subsets of of $\partial \Omega$ such that $\bigcup_{\nu} \bar{S}_{\nu}=\partial \Omega$. For each $S_{\nu}$, let $\left\{x_{1}^{(\nu)}, \ldots, x_{n}^{(\nu)}\right\}$ be a system of local coordinates, chosen so that $S_{v}$ has the representation

$$
\begin{aligned}
S_{v}=\left\{\left(x_{1}^{(v)}, \ldots, x_{n}^{(v)}\right) \in R^{n} ; x_{n}^{(v)}=F_{v}\left(x_{1}^{(v)}, \ldots, x_{n-1}^{(v)}\right),\right. \\
\\
\left.\quad\left(x_{1}^{(v)}, \ldots, x_{n-1}^{(v)}\right) \in U_{v}\right\} .
\end{aligned}
$$


If the diameters of the subsets are sufficiently small, the local coordinates can be chosen so that each $F_{v}$ satisfies

$$
\left|D^{\alpha} F_{v}(x)\right| \leqslant C, \quad x \in U_{v},
$$

where $C$ depends only on $|\alpha|$, not on $v$. Assuming this is the case, we can define

$$
\|\psi\|_{H^{k}(\partial \Omega)}^{2}=\sum_{v}\left\|\psi_{v}\right\|_{H^{k}\left(U_{v}\right)}^{2}
$$

where

$$
\psi_{v}\left(x_{1}^{(v)}, \ldots, x_{n-1}^{(v)}\right)=\psi\left(x_{1}^{(v)}, \ldots, x_{n-1}^{(v)}, F_{v}\left(x_{1}^{(v)}, \ldots, x_{n-1}^{(v)}\right)\right) .
$$

Henceforth we consider a family $\left\{\tau^{h}(\partial \Omega)\right\}_{0<h<1}$ of partitionings of $\partial \Omega$ satisfying some further conditions as given below. We let $K$ and $\lambda$ be real and $L$ an integral parameter, $K \geqslant 1, L \geqslant 1, \lambda \geqslant 0$. The subsequent conditions are assumed to hold for any given $h, 0<h<1$, and for some fixed, finite values of $K, \lambda$, and $L$.

Consider a partitioning $\tau^{h}(\partial \Omega), h$ fixed. For a set $S \subset R^{n}$, we let $d(S)$ denote the diameter of $S$. Our first assumptions are the following:

A1. (i) For all $S \in \tau^{h}(\partial \Omega), d(S) \leqslant h$.

(ii) To the partition $\tau^{h}(\partial \Omega)=\left\{S_{v}\right\}$ there corresponds a collection $\left\{C_{v}\right\}$ of spheres, $C_{v} \subset R^{n}$, such that for each $v$, the center of $C_{v}$ is in $S_{v}, d\left(C_{v}\right)=8 K d\left(S_{v}\right)$, and for each $C \in\left\{C_{v}\right\}, C \cap C_{v} \neq \varnothing$ for at most $L$ spheres $C_{v} \in\left\{C_{v}\right\}$.

Here A1(ii) may be regarded as a local regularity assumption. We will need an additional global assumption which restricts the possible global mesh refinements. To this end, let us define, for a given $S \in \tau^{h}(\partial \Omega)$, the sets $\Sigma_{k}(S)$ and $R_{k}(S)$ as follows:

$$
\begin{aligned}
& \Sigma_{0}(S)=S, \\
& \Sigma_{k}(S)=\Sigma_{k-1}(S) \cup\left\{S_{v} \in \tau^{h}(\partial \Omega) ; \bar{S}_{v} \cap \overline{\Sigma_{k-1}(S)} \neq \varnothing\right\}, \quad k=1,2, \ldots,
\end{aligned}
$$

and

$$
\begin{aligned}
& R_{0}(S)=S, \\
& R_{k}(S)=\Sigma_{k}(S)-\Sigma_{k-1}(S), \quad k=1,2, \ldots .
\end{aligned}
$$

Each $S_{\mu} \in \tau^{h}(\partial \Omega)$ is obviously a subset of $R_{k}\left(S_{v}\right), S_{v} \in \tau^{h}(\partial \Omega)$, for exactly one value of $k$. We assume:

A2. If $S_{v}, S_{\mu} \in \tau^{h}(\partial \Omega)$ and $S_{\mu} \subset R_{k}\left(S_{\nu}\right)$, then $d\left(S_{\mu}\right) / d\left(S_{\nu}\right) \leqslant K \cdot k^{\lambda}$.

In practice, $\mathrm{A} 2$ means that the refinements should be globally subexponential.

For situations where refinements of this type are used, cf. [3] .

We associate next a family of finite-dimensional subspaces $M_{2}^{h}$ to the partitionings $\tau^{h}(\partial \Omega)$. For each $h, M_{2}^{h}$ is assumed to have a basis $\left\{\psi_{1}, \ldots, \psi_{m_{h}}\right\}$ which satisfies: $k \leqslant L$.

A3. (i) For all $j, 1 \leqslant j \leqslant m_{h}, \operatorname{supp}\left\{\psi_{j}\right\} \subset \Sigma_{k}\left(S_{v}\right)$ for some $S_{v} \in \tau^{h}(\partial \Omega)$ and

(ii) If $\psi=\Sigma_{1}^{m} h \beta_{j} \psi_{j}, \beta_{j} \in R^{1}$, and $\Lambda_{v}=\left\{j ; \psi_{j} \not \equiv 0\right.$ on $\left.S_{v}\right\}, S_{v} \in \tau^{h}(\partial \Omega)$, then

$$
K \sum_{j \in \Lambda_{v}} \beta_{j}^{2}\left\|\psi_{j}\right\|_{L_{2}\left(S_{v}\right)}^{2} \geqslant\|\psi\|_{L_{2}\left(S_{v}\right)}^{2} \geqslant K^{-1} \sum_{j \in \Lambda_{v}} \beta_{j}^{2}\left\|\psi_{j}\right\|_{L_{2}\left(S_{v}\right)}^{2}
$$

In addition, we assume the following:

A4. For each $S_{v} \in \tau^{h}(\partial \Omega)$, there exists $\theta_{v} \in M_{2}^{h}$ such that $S_{v} \cap \operatorname{supp}\left\{\theta_{v}\right\} \neq \varnothing$, 
$d\left(\operatorname{supp}\left\{\theta_{v}\right\}\right) \leqslant K d\left(S_{v}\right)$, and

$$
\left|\int_{\partial \Omega} \theta_{v} d s\right|^{2} \geqslant K^{-1} d\left(S_{v}\right)^{n-1} \int_{\partial \Omega} \theta_{v}^{2} d s .
$$

Finally, let $\left\{M_{1}^{h}\right\}_{0<h<1}$ be a family of subspaces of $H^{1}(\Omega)$. We assume that for each $h$ the following compatibility conditions hold between $M_{1}^{h}$ and $M_{2}^{h}$.

A5. To the basis $\left\{\psi_{1}, \ldots, \psi_{m_{h}}\right\}$, of $M_{2}^{h}$, there corresponds the set $\left\{v_{1}, \ldots, v_{m_{h}}\right\} \subset M_{1}^{h}$ such that

(i) For any $v_{i}, 1 \leqslant i \leqslant m_{h}, v_{i} v_{j} \neq \equiv 0$ for at most $L$ functions $v_{j}, 1 \leqslant j \leqslant m_{h}$.

(ii) If $S_{v} \in \tau^{h}(\partial \Omega)$ then

$$
\left\{j ; v_{j} \not \equiv 0 \text { on } S_{v}\right\}=\left\{j ; \psi_{j} \not \equiv 0 \text { on } S_{v}\right\}=\Lambda_{v} .
$$

(iii) If $j \in \Lambda_{v}$, then

$$
K^{-1} d\left(S_{v}\right)\left|v_{j}\right|_{1, \Omega}^{2} \leqslant\left\|v_{j}\right\|_{L_{2}\left(S_{v}\right)}^{2} \leqslant K\left\|\psi_{j}\right\|_{L_{2}\left(S_{v}\right)}^{2} .
$$

(iv) If $\psi=\Sigma_{1}^{m} h \beta_{j} \psi_{j}, v=\Sigma_{1}^{m} h \beta_{j} v_{j}$, and $S_{v} \in \tau^{h}(\partial \Omega)$, then

$$
K \sum_{j \in \Lambda_{v}} \beta_{j}^{2}\left\|v_{j}\right\|_{L_{2}\left(S_{v}\right)}^{2} \geqslant\|v\|_{L_{2}\left(S_{v}\right)}^{2} \geqslant K^{-1} \sum_{j \in \Lambda_{v}} \beta_{j}^{2}\left\|v_{j}\right\|_{L_{2}\left(S_{v}\right)}^{2}
$$

and

$$
\int_{S_{v}} \psi v d s \geqslant K^{-1}\|\psi\|_{L_{2}\left(S_{v}\right)}^{2}
$$

Qualitatively, A5 imposes an upper bound for the dimension of $M_{2}^{h}$, as compared with that of the trace space of $M_{1}^{h}$ on $\partial \Omega$. In this sense A5 is a condition of the same spirit as (1.4). The difference is that A5 is only a local constraint.

3. A Stability Result. In this section, $C$ or $C_{j}$ denotes a positive constant which may take different values on different usages and may depend on $n$ and on the parameters $K, \lambda$, and $L$ of the preceding section without explicit indication. We write $C(\Omega)$ if the constant depends also on $\Omega$.

If $q \in R^{1}$, denote by $\|\cdot\|_{h, q, \partial \Omega}$ the weighted $L_{2}$-norm

$$
\|\varphi\|_{h, q, \partial \Omega}^{2}=\sum_{v} d\left(S_{v}\right)^{2 q} \int_{S_{v}} \varphi^{2} d s,
$$

where the sum is over all $S_{v} \in \tau^{h}(\partial \Omega)$. We will denote by $X_{\tau^{h}}$ the space $H^{1}(\Omega) \times$ $L_{2}(\partial \Omega)$ supplied with the norm

$$
\|(u, \varphi)\|_{X_{\tau h}}^{2}=|u|_{1, \Omega}^{2}+\|u\|_{h,-1 / 2, \partial \Omega}^{2}+\|\varphi\|_{h,-1 / 2, \partial \Omega}^{2} .
$$

In this notation, our basic stability and convergence result is as follows:

THEOREM 3.1. Let the partitionings $\tau^{h}(\Omega)$ and $\tau^{h}(\partial \Omega)$ and the spaces $M_{1}^{h} \subset$ $H^{1}(\Omega)$ and $M_{2}^{h} \subset L_{2}(\partial \Omega)$ be defined so that the conditions A1 through A5 are satisfied for some $K, \lambda$, and $L$. Further, let $f \in L_{2}(\Omega), g \in H^{r}(\partial \Omega), r>1$, and let $(u, \psi)$ be the solution of problem (1.2). Then if $h<h_{0}, h_{0}=h_{0}(\Omega) \in(0,1)$, problem (1.3) has a unique solution $\left(u_{h}, \psi_{h}\right) \in M_{1}^{h} \times M_{2}^{h}$ which satisfies

$$
\left\|\left(u_{h}, \psi_{h}\right)\right\|_{X_{\tau h}} \leqslant C(\Omega)\left(\|f\|_{0, \Omega}+\|g\|_{h,-1 / 2, \partial \Omega}\right),
$$


and

$$
\left\|(u, \psi)-\left(u_{h}, \psi_{h}\right)\right\|_{X_{\tau h}} \leqslant C \min _{(v, \varphi) \in M_{1}^{h} \times M_{2}^{h}}\|(u, \psi)-(v, \varphi)\|_{X_{\tau} h} .
$$

The proof of Theorem 3.1 will be based on the following two results.

Proposition 3.1. If $u \in H^{1}(\Omega)$ is such that

$$
\int_{\partial \Omega} u \varphi d s=0 \text { for all } \varphi \in M_{2}^{h}
$$

then if $h$ is small enough, $u$ satisfies

$$
\|u\|_{h,-1 / 2, \partial \Omega} \leqslant C|u|_{1, \Omega} .
$$

Proposition 3.2. For any $\varphi \in M_{2}^{h}$ there exists $v \in M_{1}^{h}$ such that

$$
|v|_{1, \Omega}+\|v\|_{h,-1 / 2, \partial \Omega} \leqslant 1 \text { and } \int_{\partial \Omega} v \varphi d s \geqslant C\|\varphi\|_{h, 1 / 2, \partial \Omega} .
$$

Before proving the propositions, let us show that Theorem 3.1 follows from them. For this, note first that we have

$$
\|u\|_{L_{2}(\Omega)} \leqslant C(\Omega)\left(|u|_{1, \Omega}^{2}+\|u\|_{h,-1 / 2, \partial \Omega}^{2}\right)^{1 / 2}, \quad u \in H^{1}(\Omega) .
$$

This follows, since $\|u\|_{h,-1 / 2, \partial \Omega} \geqslant d(\Omega)^{-1 / 2}\|u\|_{L_{2}(\partial \Omega)}$ and since

$$
\|u\|_{H^{1}(\Omega)} \leqslant C(\Omega)\left(|u|_{1, \Omega}^{2}+\|u\|_{L_{2}(\partial \Omega)}^{2}\right)^{1 / 2}, \quad u \in H^{1}(\Omega) .
$$

Now define on $X_{\tau} h \times X_{\tau} h$ the bilinear form

$$
B(u, \psi ; v, \varphi)=\int_{\Omega} \nabla u \cdot \nabla v d x-\int_{\partial \Omega} v \psi d s+\int_{\partial \Omega} u \varphi d s,
$$

so that Eq. (1.3) can be rewritten as

$$
B\left(u_{h}, \psi_{h}, v, \varphi\right)=-\int_{\Omega} f v d x+\int_{\partial \Omega} g \varphi d s, \quad(v, \varphi) \in M_{1}^{h} \times M_{2}^{h}
$$

The bilinear form $B$ is continuous on $X_{\tau} h \times X_{\tau} h$ :

$$
|B(u, \psi ; v, \varphi)| \leqslant\|(u, \psi)\|_{X_{\tau} h}\|(v, \varphi)\|_{X_{\tau h}} .
$$

Also, using (3.2) we have

$$
\left|-\int_{\Omega} f v d x+\int_{\partial \Omega} g \varphi d s\right| \leqslant C(\Omega)\left(\|f\|_{L_{2}(\Omega)}+\|g\|_{h,-1 / 2, \partial \Omega}\right)\|(v, \varphi)\|_{X_{\tau h}} .
$$

In view of (3.3) through (3.5), the proof of Theorem 3.1 is complete if we can show that [2, pp. 186-188]

$$
\inf _{(u, \psi) \in M_{1}^{h} \times M_{2}^{h}} \sup _{(v, \varphi) \in M_{1}^{h} \times M_{2}^{h}} \frac{B(u, \psi ; v, \varphi)}{\|(u, \psi)\|_{X_{\tau} h}\|(v, \varphi)\|_{X_{\tau h}}} \geqslant C>0 .
$$

For (3.6) to be valid in the present situation, the following two conditions are sufficient (see [4]):

$$
\begin{aligned}
u \in M_{1}^{h} & \& \int_{\partial \Omega} u \varphi d s=0 \text { for all } \varphi \in M_{2}^{h} \\
& \Rightarrow|u|_{1, \Omega}^{2} \geqslant C\left(|u|_{1, \Omega}^{2}+\|u\|_{h,-1 / 2, \partial \Omega}^{2}\right)
\end{aligned}
$$


and

$$
\inf _{\psi \in M_{2}^{h}} \sup _{v \in M_{1}^{h}} \frac{\int_{\partial \Omega} \psi v d s}{\|\psi\|_{h, 1 / 2, \partial \Omega}\left(|v|_{1, \Omega}^{2}+\|v\|_{h,-1 / 2, \partial \Omega}^{2}\right)^{1 / 2}} \geqslant C>0 .
$$

But (3.7) follows from Proposition 3.1, and (3.8) is equivalent to Proposition 3.2. Thus, if the propositions are true, the proof of Theorem 3.1 is complete.

Proof of Proposition 3.1. Let $D_{0}$ be the unit sphere of $R^{n-1}, D_{0}=\left\{x \in R^{n-1}\right.$; $|x|<1\}$, and let $E_{0}$ be the cylinder

$$
E_{0}=\left\{x=\left(z, x_{n}\right) \in R^{n} ; z \in D_{0}, 0<x_{n}<1\right\} .
$$

We prove first a preliminary result.

Lemma 3.1. Let $\delta>0$ and let $\theta \in L_{2}\left(D_{0}\right)$ be such that

$$
1=\|\theta\|_{L_{2}\left(D_{0}\right)} \leqslant \delta^{-1}\left|\int_{D_{0}} \theta(z) d z\right| .
$$

Then if $u \in H^{1}\left(E_{0}\right)$, there is a constant $C$ depending only on $\delta$ such that

$$
\int_{D_{0}}|u(z, 0)|^{2} d z \leqslant C\left\{\int_{E_{0}}|\nabla u|^{2} d x+\left|\int_{D_{0}} \theta(z) u(z, 0) d z\right|^{2}\right\} .
$$

Proof. If the assertion is not true, then there exist the sequences $\left\{u_{v}\right\}$ and $\left\{\theta_{v}\right\}$, $u_{v} \in H^{1}\left(E_{0}\right), \theta_{v} \in L_{2}\left(D_{0}\right), v=1,2, \ldots$, such that

$$
\begin{gathered}
1=\left\|\theta_{v}\right\|_{L_{2}\left(D_{0}\right)} \leqslant \delta^{-1}\left|\int_{D_{0}} \theta_{v}(z) d z\right|, \\
\left\|u_{v}\right\|_{H^{1}\left(E_{0}\right)}=1, \\
\int_{E_{0}}\left|\nabla u_{v}\right|^{2} d x+\left|\int_{D_{0}} \theta_{v}(z) u_{v}(z, 0) d z\right|^{2} \leqslant \frac{1}{v} \int_{D_{0}}\left|u_{v}(z, 0)\right|^{2} d z .
\end{gathered}
$$

By (3.10), there exists a subsequence of $\left\{u_{v}\right\}$, still called $\left\{u_{v}\right\}$, which converges in $L_{2}\left(E_{0}\right)$. Since (3.10) also implies that $\int_{D_{0}}\left|u_{v}(z, 0)\right|^{2} d z \leqslant C$, we have, by (3.11), that

$$
\int_{E_{0}}\left|\nabla u_{v}\right|^{2} d x \rightarrow 0, \quad v \rightarrow \infty .
$$

Hence, $\left\{u_{v}\right\}$ converges in $H^{1}\left(E_{0}\right)$ to a function $u_{0}(x) \equiv C_{0}=$ const. By (3.10), $C_{0} \neq 0$. However, taking the limit in (3.11) one has

$$
\begin{aligned}
0 & =\lim _{v \rightarrow \infty}\left|\int_{D_{0}} \theta_{v}(z) u_{v}(z, 0) d z\right| \\
& \geqslant \lim _{v \rightarrow \infty}\left\{\left|C_{0}\right|\left|\int_{D_{0}} \epsilon_{v}(z) d z\right|-C\left\|\theta_{v}\right\|_{L_{2}\left(D_{0}\right)}\left\|u_{v}-u_{0}\right\|_{H^{1}\left(E_{0}\right)}\right\},
\end{aligned}
$$

so that $C_{0}=0$, by (3.9), a contradiction.

Now let $\tau^{h}(\partial \Omega)$ and $M_{2}^{h}$ be given, $h$ sufficiently small so that the subsequent assumptions are valid. For each $S_{v} \in \tau^{h}(\partial \Omega)$, let $\theta_{v} \in M_{2}^{h}$ be a function referred to in $\mathrm{A} 4$, and let the sphere $C_{v} \subset R^{n}$ be as in A1(ii). By A4, if $h$ is small enough, there exist the systems of local coordinates $\left\{x_{1}^{(v)}, \ldots, x_{n}^{(v)}\right\}$, the spheres $D_{v} \subset R^{n-1}$ with center at the origin and diameter $d\left(D_{v}\right)=2 K d\left(S_{v}\right)$, and the functions $F_{v}$ defined on 
$D_{v}$ such that $S_{v} \subset \Gamma_{v}$ and $\operatorname{supp}\left\{\theta_{v}\right\} \subset \Gamma_{v}$, where

$$
\Gamma_{v}=\left\{\left(x_{1}^{(v)}, \ldots, x_{n}^{(v)}\right) \in R^{n} ; x_{n}^{(v)}=F_{v}\left(x_{1}^{(v)}, \ldots, x_{n-1}^{(v)}\right),\left(x_{1}^{(v)}, \ldots, x_{n-1}^{(v)}\right) \in \mathcal{D}_{v}\right\} .
$$

We may further assume the coordinate systems to be chosen so that each $F_{v}$ satisfies

$$
\left|D^{\alpha} F_{v}(z)\right| \leqslant C, \quad|\alpha|=1, z \in D_{v},
$$

and

$$
\left|F_{v}\left(z_{1}\right)-F_{v}\left(z_{2}\right)\right| \leqslant K d\left(S_{v}\right), \quad z_{1}, z_{2} \in D_{v} .
$$

For each $v$, define the set $E_{v}$ as

$$
\begin{aligned}
E_{v}=\left\{\left(x^{(v)}, \ldots, x_{n}^{(v)}\right)\right. & \in R^{n} ;\left(x_{1}^{(v)}, \ldots, x_{n-1}^{(v)}\right) \in D_{v} \\
& \left.F_{v}\left(x_{1}^{(v)}, \ldots, x_{n-1}^{(v)}\right)<x_{n}^{(v)}<F_{v}\left(x_{1}^{(v)}, \ldots, x_{n-1}^{(v)}\right)+K d\left(S_{v}\right)\right\} .
\end{aligned}
$$

Then if $h<h_{0}, h_{0}=h_{0}(\Omega)$ sufficiently small, we have $E_{v} \subset \Omega$ for each $v$. From (3.12) and from Al(ii) it is also easily verified that

$$
E_{v} \subset C_{v} \cap \Omega, \quad v=1,2, \ldots
$$

The set $E_{v}$ may be mapped onto the cylinder $E_{0}$ of Lemma 3.1 by the mapping $x \rightarrow \hat{x}=J_{v}(x)$ defined as

$$
\begin{aligned}
& \hat{x}_{i}=K^{-1} d\left(S_{v}\right)^{-1} x_{i}^{(v)}, \quad i=1, \ldots, n-1, \\
& \hat{x}_{n}=K^{-1} d\left(S_{v}\right)^{-1}\left[x_{n}^{(v)}-F_{v}\left(x_{1}^{(v)}, \ldots, x_{n-1}^{(v)}\right)\right] .
\end{aligned}
$$

By the properties of $F_{v}$ assumed above, $J_{v}: E_{v} \rightarrow E_{0}$ is an invertible smooth transformation. Moreover, we obtain from the definition of $J_{v}$ the formula

$$
\int_{\Gamma_{\nu}} \varphi(s) d s=\int_{D_{0}} e_{v}(z) \hat{\varphi}_{v}(z) d z, \quad \varphi \in L_{2}\left(\Gamma_{v}\right)
$$

where $\hat{\varphi}_{v}(z)=\varphi\left(J_{v}^{-1}(z, 0)\right)$ and $e_{v}$ satisfies

$$
C_{1} K^{n-1} d\left(S_{v}\right)^{n-1} \leqslant e_{v}(z) \leqslant C_{2} K^{n-1} d\left(S_{v}\right)^{n-1}, \quad z \in \mathcal{D}_{0}
$$

Now let $u \in H^{1}(\Omega)$ be such that (3.1) holds, let $u_{v}$ be the function $u$ in the coordinate system $\left\{x_{1}^{(v)}, \ldots, x_{n}^{(v)}\right\}$, and let $\hat{u}_{v}(x)=u_{v}\left(J_{v}^{-1}(x)\right), x \in E_{0}$. Then, since $\Gamma_{v} \supset$ $\operatorname{supp}\left\{\theta_{v}\right\}$, we have, using (3.1) and (3.14),

$$
0=\int_{\Gamma_{v}} u_{v} \theta_{v} d s=\int_{D_{0}} e_{v}(z) \hat{\theta}_{v}(z) \hat{u}_{v}(z, 0) d z
$$

where $\hat{\theta}_{v}(z)=\theta_{v}\left(J_{v}^{-1}(z, 0)\right)$. By A4 we have

or

$$
\left|\int_{\Gamma_{v}} \theta_{v} d s\right|^{2} \geqslant K^{-1} d\left(S_{v}\right)^{n-1} \int_{\Gamma_{v}}\left|\theta_{v}\right|^{2} d s
$$

$$
\left|\int_{D_{0}} e_{v} \hat{\theta}_{v} d z\right|^{2} \geqslant K^{-1} d\left(S_{v}\right)^{n-1} \int_{D_{0}} e_{v}\left|\hat{\theta}_{v}\right|^{2} d z
$$

or, using (3.15),

$$
\left|\int_{D_{0}} e_{v} \hat{\theta}_{v} d z\right|^{2} \geqslant C \int_{D_{0}}\left|e_{v} \hat{\theta}_{v}\right|^{2} d z
$$


Let $\theta_{v}$ be normalized so that $\left\|e_{v} \hat{\theta}_{v}\right\|_{L_{2}\left(D_{0}\right)}=1$. Then, choosing $\theta=e_{v} \hat{\theta}_{v}$ in Lemma 3.1 , we conclude from (3.16) and (3.17) and from the lemma that

$$
\int_{D_{0}}\left|\hat{u}_{v}(z, 0)\right|^{2} d z \leqslant C \int_{E_{0}}\left|D^{1} \hat{u}_{v}\right|^{2} d x
$$

or, in the original coordinates,

$$
d\left(S_{v}\right)^{-1} \int_{\Gamma_{v}}\left|u_{v}\right|^{2} d s \leqslant C \int_{E_{v}}\left|D^{1} u_{v}\right|^{2} d x
$$

We sum the last inequality over $v$ and use (3.13), together with A1(ii), to finally obtain

$$
\begin{aligned}
\|u\|_{h,-1 / 2, \partial \Omega}^{2} & \leqslant \sum_{v} d\left(S_{v}\right)^{-1} \int_{\Gamma_{v}}\left|u_{v}\right|^{2} d s \\
& \leqslant C \sum_{v} \int_{E_{v}}\left|D^{1} u_{v}\right|^{2} d x \leqslant C L \int_{\Omega}\left|D^{1} u\right|^{2} d x .
\end{aligned}
$$

This completes the proof.

Proof of Proposition 3.2. Let $V^{h}$ denote the subspace of $L_{2}(\partial \Omega)$ spanned by the functions $v_{i \mid \partial \Omega}, i=1, \ldots, m_{h}, v_{i}$ as in A5. It is easily verified from A5 that $\operatorname{dim}\left(V^{h}\right)=m_{h}$, i.e., $V^{h}$ and $M_{2}^{h}$ are of equal dimension. We also recall from A5(i), (iii), and (iv) that if $v=\Sigma_{1}^{m} h \beta_{j} v_{j}$, then

$$
\begin{aligned}
|v|_{1, \Omega}^{2} & \leqslant L \sum_{1}^{m_{h}} \beta_{j}^{2}\left|v_{j}\right|_{1, \Omega}^{2} \\
& \leqslant L K \sum_{v} d\left(S_{v}\right)^{-1} \sum_{j \in \Lambda_{v}} \beta_{j}^{2}\left\|v_{j}\right\|_{L_{2}\left(S_{v}\right)}^{2} \leqslant L K^{2}\|v\|_{h,-1 / 2, \partial \Omega}^{2} .
\end{aligned}
$$

In view of this, the assertion of Proposition 3.2 follows if we can show that

$$
\inf _{\psi \in M_{2}^{h}} \sup _{v \in V} \frac{\int_{\partial \Omega} \psi \varphi d s}{\|\psi\|_{h, 1 / 2, \partial \Omega}\|v\|_{h,-1 / 2, \partial \Omega}} \geqslant C>0 .
$$

The remaining problem of proving (3.18) can be further modified as follows. First, define the linear mapping $P_{h}: L_{2}(\partial \Omega) \rightarrow M_{2}^{h}$ as

$$
P_{h} \psi \in M_{2}^{h}: b\left(P_{h} \psi, v\right)=b(\psi, v) \quad \forall v \in V^{h},
$$

where

$$
b(\psi, \varphi)=\int_{\partial \Omega} \psi \varphi d s, \quad \psi, \varphi \in L_{2}(\partial \Omega)
$$

Then we have

Lemma 3.2. (3.18) holds, provided that $P_{h}$ satisfies

$$
\left\|P_{h} \varphi\right\|_{h, 1 / 2, \partial \Omega} \leqslant C\|\varphi\|_{h, 1 / 2, \partial \Omega}, \quad \varphi \in L_{2}(\partial \Omega) .
$$

Proof. Denote by $H_{1}$ and $H_{2}$ the Hilbert spaces obtained by supplying $L_{2}(\partial \Omega)$ with the inner products

$$
(\psi, \varphi)_{H_{1}}=\sum_{v} d\left(S_{v}\right) \int_{S_{v}} \psi \varphi d s, \quad \text { and } \quad(\psi, \varphi)_{H_{2}}=\sum_{v} d\left(S_{v}\right)^{-1} \int_{S_{v}} \psi \varphi d s
$$

respectively, where the sums are over $S_{v} \in \tau^{h}(\partial \Omega)$. Then $\|\cdot\|_{H_{1}}=\|\cdot\|_{h, 1 / 2, \partial \Omega}$, and 
$\|\cdot\|_{H_{2}}=\|\cdot\|_{h,-1 / 2, \partial \Omega}$, and $b$ is a bounded bilinear form on $H_{1} \times H_{2}$ :

$$
|b(\psi, \varphi)| \leqslant\|\psi\|_{H_{1}}\|\varphi\|_{H_{2}}, \quad \psi \in H_{1}, \varphi \in H_{2} .
$$

We also easily contend that

$$
\inf _{\psi \in H_{1}} \sup _{\varphi \in H_{2}} \frac{b(\psi, \varphi)}{\|\psi\|_{H_{1}}\|\varphi\|_{H_{2}}}=\inf _{\varphi \in H_{2}} \sup _{\psi \in H_{1}} \frac{b(\psi, \varphi)}{\|\psi\|_{H_{1}}\|\varphi\|_{H_{2}}}=1 .
$$

Assume that (3.21) holds. Then, using (3.23), (3.19), and (3.21) we find that if $v \in V^{h}$, then

$$
\begin{aligned}
\|v\|_{H_{2}} & =\sup _{\psi \in H_{1}} \frac{b(\psi, v)}{\|\psi\|_{H_{1}}}=\sup _{\psi \in H_{1}} \frac{b\left(P_{h} \psi, v\right)}{\|\psi\|_{H_{1}}} \\
& \leqslant C \sup _{\psi \in H_{1}} \frac{b\left(P_{h} \psi, v\right)}{\left\|P_{h} \psi\right\|_{H_{1}}} \leqslant C \sup _{\psi \in M_{2}^{h}} \frac{b(\psi, v)}{\|\psi\|_{H_{1}}}, \quad v \in V^{h} .
\end{aligned}
$$

Proceeding as in [2, p. 113], we now define the linear mapping $E_{h}: V^{h} \rightarrow M_{2}^{h}$ through the formula

$$
b(\psi, v)=\left(\psi, E_{h} v\right)_{H_{1}}, \quad \psi \in M_{2}^{h}, v \in V^{h} .
$$

Then, by (3.22) and (3.24), $E_{h}$ is bounded and 1:1 [2, pp. 113-115]. Moreover, as $M_{2}^{h}$ and $V^{h}$ are of equal dimension, $E_{h}$ is onto $M_{2}^{h}$. Then if $E_{h}^{*}: M_{2}^{h} \rightarrow V^{h}$ is the adjoint mapping defined as

$$
b(\psi, v)=\left(E_{h}^{*} \psi, v\right)_{H_{2}}, \quad \psi \in M_{2}^{h}, v \in V^{h},
$$

we have, using (3.24) and a well-known argument (cf. [2, pp. 112-115] or [4]), that

$$
\begin{aligned}
\inf _{\psi \in M_{2}^{h}} \sup _{v \in V^{h}} \frac{b(\psi, v)}{\|\psi\|_{H_{1}}\|v\|_{H_{2}}} & =\left\|\left(E_{h}^{*}\right)^{-1}\right\|^{-1}=\left\|E_{h}^{-1}\right\|^{-1} \\
& =\inf _{v \in V^{h}} \sup _{\psi \in M_{2}^{h}} \frac{b(\psi, v)}{\|\psi\|_{H_{1}}\|v\|_{H_{2}}} \geqslant C>0 .
\end{aligned}
$$

This proves the lemma.

To prove (3.21), let $\varphi \in L_{2}(\partial \Omega)$ be given and define, for $S_{v} \in \tau^{h}(\partial \Omega)$, the function $\varphi_{\nu}$ as

$$
\begin{array}{rlrl}
\varphi_{v}(x) & =\varphi(x), & & x \in S_{v}, \\
& =0, & x \in \partial \Omega \backslash S_{v} .
\end{array}
$$

With the sets $R_{k}\left(S_{v}\right), k=0,1,2, \ldots$, defined as in (2.6), let us first prove that $P_{h} \varphi_{\nu}(x)$ decays exponentially in the following sense:

$$
\left\|P_{h} \varphi_{v}\right\|_{L_{2}\left(R_{k}\left(S_{v}\right)\right)} \leqslant C e^{-\alpha k}\left\|\varphi_{v}\right\|_{L_{2}\left(S_{v}\right)}, \quad \alpha>0
$$

where $\alpha$ does not depend on $h$.

Note that, in the case of a quasiuniform partitioning, a result analogous to (3.25) was proved in [6] in a different context. 
We start the proof of (3.25) by first introducing a real parameter $q, q>1$, and defining

$$
\tilde{v}_{j}(x)=q^{m_{j}} v_{j}(x), \quad j=1, \ldots, m_{h},
$$

where $m_{j}=0$ if $\operatorname{supp}\left\{v_{j}\right\} \cap S_{v} \neq \varnothing$, and otherwise $m_{j}$ is chosen so that $\operatorname{supp}\left\{v_{j}\right\} \cap$ $\Sigma_{m_{j}-1}\left(S_{v}\right)=\varnothing$ and $\operatorname{supp}\left\{v_{j}\right\} \cap \Sigma_{m_{j}}\left(S_{v}\right) \neq \varnothing$, where the sets $\Sigma_{k}\left(S_{v}\right)$ are as in (2.5).

Consider a given subset $S_{\mu} \in \tau^{h}(\partial \Omega)$ such that $S_{\mu} \subset R_{k}\left(S_{\nu}\right), k \geqslant 1$. By the assumptions A3(i) and A5(ii), if $v_{j} \neq \equiv 0$ on $S_{\mu}$, then $\operatorname{supp}\left\{v_{j}\right\} \cap \partial \Omega \subset \Sigma_{L}\left(S_{\mu}\right)$.

In view of (2.5) and the above definition of $m_{j}$, this implies that $k-L \leqslant m_{j} \leqslant k$, and therefore, by (3.26),

$$
\tilde{v}_{j}(x)-q^{k} v_{j}(x)=q^{k} \Delta_{j} v_{j}(x), \quad\left|\Delta_{j}\right| \leqslant 1-q^{-L} .
$$

Now let $P_{h} \varphi_{v}$ be expanded as $P_{h} \varphi_{\nu}=\Sigma_{1}^{m} h \beta_{j} \psi_{j}$, and let $\tilde{v}=\Sigma_{1}^{m} h \beta_{j} \tilde{v}_{j}$. Then if $\Lambda_{\mu}$ is as in A5(ii), we get, using A3(ii), A5(iii), A5(iv) and (3.27),

$$
\begin{aligned}
& q^{-k} \int_{S_{\mu}}\left(P_{h} \varphi_{\nu}\right) \tilde{v} d s \\
&=\int_{S_{\mu}}\left(P_{h} \varphi_{v}\right)\left(\sum_{j \in \Lambda_{\mu}} \beta_{j} v_{j}\right) d s+\int_{S_{\mu}}\left(P_{h} \varphi_{v}\right)\left(\sum_{j \in \Lambda_{\mu}} \Delta_{j} \beta_{j} v_{j}\right) d s \\
& \geqslant {\left[K^{-1}-1 / 2\left(1-q^{-L}\right)\right]\left\|P_{h} \varphi_{\nu}\right\|_{L_{2}\left(S_{\mu}\right)}^{2} } \\
&-\frac{1}{2\left(1-q^{-L}\right)} \int_{S_{\mu}}\left(\sum_{j \in \Lambda_{\mu}} \Delta_{j} \beta_{j} v_{j}\right)^{2} d s \\
& \geqslant {\left[K^{-1}-1 / 2\left(1-q^{-L}\right)\right]\left\|P_{h} \varphi_{\nu}\right\|_{L_{2}}^{2}\left(S_{\mu}\right) } \\
&-1 / 2 K\left(1-q^{-L}\right) \sum_{j \in \Lambda_{\mu}} \beta_{j}^{2} \int_{S_{\mu}} v_{j}^{2} d s \\
& \geqslant {\left[K^{-1}-1 / 2\left(K^{3}+1\right)\left(1-q^{-L}\right)\right]\left\|P_{h} \varphi_{\nu}\right\|_{L_{2}\left(S_{\mu}\right)}^{2} . }
\end{aligned}
$$

Taking $q$ sufficiently close to (but still larger than) unity, we can have

$$
K^{-1}-1 / 2\left(K^{3}+1\right)\left(1-q^{-L}\right)>1 / 2 K^{-1} \text {. }
$$

Upon summing over $\mu,(3.28)$ and (3.29) then imply

$$
\begin{aligned}
\int_{\partial \Omega}\left(P_{h} \varphi_{v}\right) \tilde{v} d s & =\sum_{k} \int_{R_{k}\left(S_{v}\right)}\left(P_{h} \varphi_{v}\right) \tilde{v} d s \\
& \geqslant 1 / 2 K^{-1} \sum_{k} q^{k} \int_{R_{k}\left(S_{v}\right)}\left(P_{h} \varphi_{v}\right)^{2} d s
\end{aligned}
$$

On the other hand, we find from the definition of $\tilde{v}$ and from A3(ii), A5(iii), and A5(iv) that

$$
\int_{S_{v}} \varphi_{v} \tilde{v} d s \leqslant K^{3 / 2}\left\|\varphi_{v}\right\|_{L_{2}\left(S_{v}\right)}\left\|P_{h} \varphi_{v}\right\|_{L_{2}\left(S_{v}\right)}
$$


Recalling that $\tilde{v} \in V^{h}$, we then have, using (3.19) and (3.20),

$$
\begin{aligned}
\int_{\partial \Omega}\left(P_{h} \varphi_{v}\right) \tilde{v} d s & =\int_{\partial \Omega} \varphi_{v} \tilde{v} d s=\int_{S_{v}} \varphi_{v} \tilde{v} d s \\
& \leqslant K^{3 / 2}\left\|\varphi_{v}\right\|_{L_{2}\left(S_{v}\right)}\left\|P_{h} \varphi_{v}\right\|_{L_{2}\left(S_{v}\right)} \\
& \leqslant K^{3 / 2}\left\|\varphi_{v}\right\|_{L_{2}\left(S_{v}\right)}\left\{\sum_{k} q^{k}\left\|P_{h} \varphi_{v}\right\|_{L_{2}\left(R_{k}\left(S_{v}\right)\right)}^{2}\right\}^{1 / 2}
\end{aligned}
$$

Since $q>1$, the estimate (3.25) follows from (3.30) and (3.31).

Combining (3.25) with the assumption A2, we get for all $S_{v}, S_{\mu} \in \tau^{h}(\partial \Omega)$ the estimate

$$
\begin{aligned}
& d\left(S_{\mu}\right) \int_{S_{\mu}}\left(P_{h} \varphi_{\nu}\right)^{2} d s \leqslant d\left(S_{\mu}\right) \int_{R_{k}\left(S_{v}\right)}\left(P_{h} \varphi_{\nu}\right)^{2} d s \\
& \leqslant C k^{\lambda} e^{-\alpha k} d\left(S_{\nu}\right) \int_{S_{\nu}} \varphi_{\nu}^{2} d s \leqslant C_{1} e^{-\alpha_{1} k} d\left(S_{\nu}\right) \int_{S_{v}} \varphi_{\nu}^{2} d s
\end{aligned}
$$

where $k$ is such that $S_{\mu} \subset R_{k}\left(S_{v}\right)$, and $0<\alpha_{1}<\alpha$.

Finally, to estimate $P_{h} \varphi$, write $P_{h} \varphi=\Sigma_{v} P_{h} \varphi_{v}$, and apply the inequality

$$
\left(\sum_{1}^{\infty} \alpha_{k}\right)^{2} \leqslant C \sum_{1}^{\infty} e^{\beta k} \alpha_{k}^{2}, \quad \beta>0, C=C(\beta), \alpha_{k} \in R^{1},
$$

together with (3.32) to first get

$$
\begin{aligned}
& d\left(S_{\mu}\right) \int_{S_{\mu}}\left(P_{h} \varphi\right)^{2} d s \\
& \quad=d\left(S_{\mu}\right) \int_{S_{\mu}}\left[\sum_{k}\left(\sum_{\nu \in \Lambda_{k, \mu}} P_{h} \varphi_{\nu}\right)\right]^{2} d s \\
& \quad \leqslant C d\left(S_{\mu}\right) \sum_{k} e^{\beta k} \int_{S_{\mu}}\left(\sum_{\nu \in \Lambda_{k, \mu}} P_{h} \varphi_{\nu}\right)^{2} d s \\
& \quad \leqslant C_{1} d\left(S_{\mu}\right) \sum_{k} N_{k, \mu} e^{\beta k} \sum_{v \in \Lambda_{k, \mu}} \int_{S_{\mu}}\left(P_{h} \varphi_{v}\right)^{2} d s \\
& \quad \leqslant C_{2} \sum_{k} e^{\left(\beta-\alpha_{1}\right) k} N_{k, \mu} \sum_{\nu \in \Lambda_{k, \mu}} d\left(S_{v}\right) \int_{S_{v}} \varphi_{\nu}^{2} d s,
\end{aligned}
$$

where $\Lambda_{k, \mu}=\left\{v ; S_{v} \subset R_{k}\left(S_{\mu}\right)\right\}$, and $N_{k, \mu}$ denotes the number of sets $S_{v} \in \tau^{h}(\partial \Omega)$ which are contained in $R_{k}\left(S_{\mu}\right)$.

$N_{k, \mu}$ can be estimated as follows. First, if $C_{v}$ is the sphere associated to $S_{v}$ as in $\mathrm{A} 1$ (ii), then, by $\mathrm{A} 1$ (ii) and $\mathrm{A} 2$, the set $\bigcup_{v \in \Lambda_{k, \mu}} C_{v}$ is contained in a sphere of radius

$$
\rho \leqslant K d\left(S_{\mu}\right) \sum_{j=0}^{k} j^{\lambda}+4 K\left(K d\left(S_{\mu}\right) k^{\lambda}\right) \leqslant C d\left(S_{\mu}\right) k^{\lambda+1} .
$$

Second, by $\mathrm{A} 1$ (ii) and $\mathrm{A} 2$,

$$
d\left(C_{\nu}\right) \geqslant 8 K\left[K^{-1} k^{-\lambda} d\left(S_{\mu}\right)\right], \quad v \in \Lambda_{k, \mu} .
$$


Therefore, using (3.34), (3.35), and A1(ii), we have the estimate

$$
L\left[C d\left(S_{\mu}\right) k^{\lambda+1}\right]^{n} \geqslant N_{k, \mu}\left[4 k^{-\lambda} d\left(S_{\mu}\right)\right]^{n}
$$

so that

$$
N_{k, \mu}<C k^{n(2 \lambda+1)} \text {. }
$$

Upon taking $0<\beta<\alpha_{1}$ in (3.33) and using (3.36), we get

$$
d\left(S_{\mu}\right) \int_{S_{\mu}}\left(P_{h} \varphi\right)^{2} d s \leqslant C \sum_{k} e^{-\alpha_{2} k} \sum_{\nu \in \Lambda_{k, \mu}} d\left(S_{v}\right) \int_{S_{\nu}} \varphi^{2} d s,
$$

with $\alpha_{2}>0$. Summing this over $\mu$ and again using (3.36), we finally have

$$
\begin{aligned}
\left\|P_{h} \varphi\right\|_{h, 1 / 2, \partial \Omega}^{2} & \leqslant C \sum_{v} \sum_{k} \sum_{\mu \in \Lambda_{k, v}} e^{-\alpha_{2} k} d\left(S_{v}\right) \int_{S_{v}} \varphi^{2} d s \\
& =C \sum_{v}\left(\sum_{k} e^{-\alpha_{2} k} N_{k, v}\right) d\left(S_{v}\right) \int_{S_{v}} \varphi^{2} d s \\
& \leqslant C_{1} \sum_{v}\left(\sum_{k=1}^{\infty} e^{-\alpha_{2} k} k^{n(2 \lambda+1)}\right) d\left(S_{v}\right) \int_{S_{v}} \varphi^{2} d s \\
& \leqslant C_{2}\|\varphi\|_{h, 1 / 2, \partial \Omega}^{2} .
\end{aligned}
$$

This proves (3.21), and the proof of Proposition 3.2 is complete.

We point out that the regularity properties of $\partial \Omega$ did not play any explicit role in the proof of Proposition 3.2. Also, Proposition 3.1 is true under weaker regularity assumptions on $\Omega$; for example, it would suffice to assume that $\Omega$ is a Lipschitzian domain with piecewise smooth boundary. Theorem 3.1 is then true also for such more general domains.

4. Application. Let $\left\{\tau^{h}(\partial \Omega)\right\}_{0<h<1}$ be a family of partitionings of $\partial \Omega$ satisfying assumptions $\mathrm{A} 1$ and $\mathrm{A} 2$. Let $h$ be fixed sufficiently small and assume that for each $S_{v} \in \tau^{h}(\partial \Omega)$ the systems of local coordinates $\left\{x_{1}^{(v)}, \ldots, x_{n}^{(v)}\right\}$ are defined so that Eqs. (2.1) through (2.4) are satisfied.

Let $k$ be a fixed integer, $k \geqslant 1$. We define $M_{2}^{h}$ as the maximal subspace of $L_{2}(\partial \Omega)$ such that if $\psi \in M_{2}^{h}, S_{v} \in \tau^{h}(\partial \Omega)$, and $\psi_{v}$ is defined as in (2.4), then $\psi_{v}$ is a polynomial of degree $k-1$ in the variables $x_{1}^{(v)}, \ldots, x_{n-1}^{(v)}$.

To define the interior subspaces $M_{1}^{h}$, consider a family of partitionings $\pi^{h}$ of $R^{n}$ into $n$-simplicial subdomains $t_{v}$. We assume the partitionings to be arranged in the usual way so that any face of any $t \in \pi^{h}$ is also a face of another $t_{v} \in \pi^{h}$. The following additional assumptions will be made:

B1. (i) $t \in \pi^{h} \Rightarrow d(t) \leqslant h$.

(ii) For each $t \in \pi^{h}$, either $t \cap \Omega=\varnothing$ or $t \cap \Omega$ contains a sphere of radius $\rho \geqslant$ $K^{-1} d(t)$.

(iii) If $t \in \pi^{h}, S \in \tau^{h}(\partial \Omega)$, and $t \cap S \neq \varnothing$, then $K^{-1} d(S) \leqslant d(t) \leqslant K d(S)$. 
We note that if $t \in \Omega$, then (ii) reduces to the ordinary regularity assumption $[5]$.

We let $\left\{\tau^{h}(\Omega)\right\}_{0<h<1}$ be a family of partitionings of $\Omega$, defined as

$$
\tau^{h}(\Omega)=\left\{T_{v} ; T_{v}=t_{\mu} \cap \Omega, t_{\mu} \in \pi^{h}, t_{\mu} \cap \Omega \neq \varnothing\right\} .
$$

We will further denote by $\Omega_{h}$ the polyhedron

$$
\Omega_{h}=\bigcup_{t_{v} \in \pi_{;} t_{v} \cap \Omega \neq \varnothing} \overline{t_{v}} .
$$

For each $h$, let $M_{1}^{h}$ be defined as the maximal subspace of $H^{1}(\Omega)$ such that, for each $T \in \tau^{h}(\Omega),\left.M_{1}^{h}\right|_{T}$ equals the space of polynomials of order $k$ on $T$, where $k$ has the same value as in the definition of $M_{2}^{h}$.

In order to be able to verify the conditions A3 through A5 for the above subspaces, a further constraint on the partitionings will be required. In particular, A5 is not generally true unless the partitioning $\tau^{h}(\partial \Omega)$ is locally by a certain factor coarser than the partitioning induced by $\tau^{h}(\Omega)$ on $\partial \Omega$. A sufficient condition can be found as follows. Let $\left\{y_{1}, \ldots, y_{p_{h}}\right\}$ be the set of vertices of the simplices $t_{v} \in \pi^{h}$ which are located on $\partial \Omega_{h}$. For each $i, 1 \leqslant i \leqslant p_{h}$, let

$$
E_{i}^{h}=\bigcup_{\nu \in \Lambda_{i}} \overline{t_{v}}
$$

where $\Lambda_{i}=\left\{v ; t_{v} \in \pi^{h}, y_{i} \in \overline{t_{v}}\right\}$. Then it suffices to assume

B2. Each $S_{v} \in \tau^{h}(\partial \Omega)$ contains at least one of the sets $\partial \Omega \cap E_{i}^{h}, 1 \leqslant i \leqslant p_{h}$.

To verify the assumptions of Section 2, we need first a basis for $M_{2}^{h}$. For $S_{v} \in$ $\tau^{h}(\partial \Omega)$, let $U_{v}$ be as in (2.1), and let $C_{0}>0$ be a fixed constant. Then, by a scaling argument, there exists for each $v$ a basis $\left\{\varphi_{1}^{(v)}, \ldots, \varphi_{m}^{(v)}\right\}$ of the space of polynomials of degree $\leqslant k-1$ in $R^{n-1}$ such that if $D_{i} \subset R^{n-1}, i=1,2,3$, are any spheres satisfying $D_{i} \cap U_{v} \neq \varnothing$ and $C_{0} d\left(S_{v}\right) \geqslant d\left(D_{i}\right) \geqslant C_{0}^{-1} d\left(S_{v}\right)$, then

$$
\begin{aligned}
C^{-1} \sum_{i=1}^{m} \beta_{i}^{2}\left\|\varphi_{i}^{(v)}\right\|_{L_{2}\left(D_{1}\right)}^{2} & \leqslant \int_{D_{2}}\left(\sum_{i=1}^{m} \beta_{i} \varphi_{i}^{(v)}\right)^{2} d s \\
& \leqslant C \sum_{i=1}^{m} \beta_{i}^{2}\left\|\varphi_{i}^{(v)}\right\|_{L_{2}\left(D_{3}\right)}^{2}, \quad \beta_{i} \in R^{1},
\end{aligned}
$$

where $C$ depends only on $C_{0}, k$, and $n$. Assuming such bases to be defined, we take for the basis of $M_{2}^{h}$ the set $\bigcup_{\nu}\left\{\psi_{1}^{(\nu)}, \ldots, \psi_{m}^{(\nu)}\right\}$, where

$$
\begin{aligned}
\psi_{i}^{(v)}(x) & =\varphi_{i}^{(v)}\left(x_{1}^{(v)}, \ldots, x_{n-1}^{(v)}\right), & & x \in S_{v}, \\
& =0, & & x \in \partial \Omega \backslash S_{v} .
\end{aligned}
$$

For the above basis, A3(i) is true with $L=0$. To verify A3(ii), note that, by B2, B1(ii), B1(iii), (2.1), and (2.2), if $h$ is sufficiently small, then each $U_{v}$ in (2.1) contains a sphere of radius $\rho>C d\left(S_{v}\right), C>0, C$ independent of $v$. Denote this sphere by 
$D_{v, 1}$, and let $D_{v, 2}$ be a sphere of radius $\rho=d\left(S_{v}\right)$ such that $D_{v, 2} \supset U_{v}$. Then the validity of $\mathrm{A} 3(\mathrm{ii})$, for some constant $K$, is obvious from the following chain of inequalities:

$$
\begin{aligned}
& \sum_{i=1}^{m} \beta_{i}^{2}\left\|\psi_{i}^{(v)}\right\|_{L_{2}\left(S_{v}\right)}^{2} \geqslant C_{1} \sum_{i=1}^{m} \beta_{i}^{2} l i \varphi_{i}^{(v)} \|_{L_{2}\left(D_{v, 1}\right)}^{2} \\
& \geqslant C_{2} \sum_{i=1}^{m} \beta_{i}^{2}\left\|\varphi_{i}^{(v)}\right\|_{L_{2}\left(D_{v, 2}\right)}^{2} \geqslant C_{3}\left\|\sum_{i=1}^{m} \beta_{i} \varphi_{i}^{(v)}\right\|_{L_{2}\left(D_{v, 2}\right)}^{2} \\
& \geqslant C_{4}\left\|\sum_{i=1}^{m} \beta_{i} \psi_{i}^{(v)}\right\|_{L_{2}\left(S_{v}\right)}^{2} \geqslant C_{5}\left\|\sum_{i=1}^{m} \beta_{i} \varphi_{i}^{(v)}\right\|_{L_{2}\left(D_{v, 1}\right)}^{2} \\
& \geqslant C_{6} \sum_{i=1}^{m} \beta_{i}^{2}\left\|\varphi_{i}^{(v)}\right\|_{L_{2}\left(D_{v, 1}\right)}^{2} \geqslant C_{7} \sum_{i=1}^{m} \beta_{i}^{2}\left\|\varphi_{i}^{(v)}\right\|_{L_{2}\left(D_{v, 2}\right)}^{2} \\
& \geqslant C_{8} \sum_{i=1}^{m} \beta_{i}^{2}\left\|\psi_{i}^{(v)}\right\|_{L_{2}\left(S_{v}\right)}^{2}, \quad \beta_{i} \in R^{1} .
\end{aligned}
$$

Here the constants $C_{j}$ are positive and independent of $\nu$; the second, third, sixth, and seventh inequalities follow from (4.2) and the remaining ones from (4.3), (2.1), (2.2), and from the inclusions $D_{v, 1} \subset U_{v} \subset D_{v, 2}$.

Assumption A4 is easily verified. Taking

$$
\begin{aligned}
\theta_{v}(x) & =1, & & x \in S_{v}, \\
& =0, & & x \in \partial \Omega \backslash S_{v},
\end{aligned}
$$

it suffices to verify that, for all $S_{v} \in \tau^{h}(\partial \Omega)$,

$$
\int_{S_{v}} d s \geqslant C d\left(S_{v}\right)^{n-1}
$$

with $C$ independent of $v$. This follows from B2, B1(ii), and $\mathrm{B} 1$ (iii).

Finally, A5 should be verified. To this end, let $\left\{\xi_{1}, \ldots, \xi_{p_{h}}\right\}$ be the set of continuous functions in $R^{n}$ such that for each $i, 1 \leqslant i \leqslant p_{h}, \xi_{i}$ is a polynomial of degree one in each $t_{v} \in \pi^{h},\left\|\xi_{i}\right\|_{L_{\infty}\left(R^{n}\right)}=1, \xi_{i}(x) \geqslant 0$, and $\operatorname{supp}\left\{\xi_{i}\right\}=E_{i}^{h}$, where $E_{i}^{h}$ is as in (4.1). As is well known, such functions exist. Then define

$$
v_{j}^{(v)}(x)=\xi_{i_{v}}\left(x_{1}^{(v)}, \ldots, x_{n}^{(v)}\right) \varphi_{j}^{(v)}\left(x_{1}^{(v)}, \ldots, x_{n-1}^{(v)}\right), \quad 1 \leqslant j \leqslant m, x \in \Omega,
$$

where $\left\{x_{1}^{(v)}, \ldots, x_{n}^{(v)}\right\}$ and $\left\{\varphi_{1}^{(v)}, \ldots, \varphi_{m}^{(v)}\right\}$ are as above, and $i_{v}$ is chosen so that

$$
S_{v} \supset E_{i_{v}}^{h} \cap \partial \Omega .
$$

Functions $v_{j}^{(v)}$ are continuous and piecewise polynomials of degree $k$, so $v_{j}^{(v)} \in M_{1}^{h}$. Now let us verify that the set $\bigcup_{\nu}\left\{v_{1}^{(v)}, \ldots, v_{m}^{(v)}\right\}$ is what we are looking for. First, A5(i) follows from the assumed regularity property B1(ii). Second, A5(ii) is true since $\operatorname{supp}\left\{v_{j}^{(v)}\right\} \cap \partial \Omega \subset S_{v}=\operatorname{supp}\left\{\psi_{j}^{(v)}\right\}$. Third, A5(iii) follows from a scaling argument, using $\mathrm{B} 1$ (ii) and (4.2).

Finally, to verify A5(iv), note that if $h$ is small enough, then (2.1), (2.2), Bl(ii), and Bl (iii) imply the existence of the spheres $D_{v} \subset R^{n-1}$, with $d\left(D_{v}\right) \geqslant C d\left(S_{v}\right), C$ independent of $v$, such that $D_{v} \subset U_{v}$ and so that $\xi_{i_{v}}$ satisfies 


$$
\xi_{i_{v}}(x) \geqslant C>0, x \in \Delta_{v}=\left\{\left(x_{1}^{(v)}, \ldots, x_{n}^{(v)}\right) \in \partial \Omega ; x_{1}^{(v)}, \ldots, x_{n-1}^{(v)} \in D_{v}\right\} .
$$

Then, since $\xi_{i_{v}}$ is everywhere nonnegative, we get, using (2.1), (2.2), (4.2), and (4.4) through (4.6),

$$
\begin{gathered}
\int_{S_{v}}\left(\sum_{1}^{m} \beta_{j} v_{j}^{(v)}\right)\left(\sum_{1}^{m} \beta_{j} \psi_{j}^{(v)}\right) d s \geqslant \int_{\Delta_{v}} \xi_{i_{v}}\left(\sum_{1}^{m} \beta_{j} \psi_{j}^{(v)}\right)^{2} d s \\
\geqslant C \int_{D_{v}}\left(\sum_{1}^{m} \beta_{j} \varphi_{j}^{(v)}\right)^{2} d x_{1}^{(v)} \ldots d x_{n-1}^{(v)} \\
\geqslant C_{1} \int_{U_{v}}\left(\sum_{1}^{m} \beta_{j} \varphi_{j}^{(v)}\right)^{2} d x_{1}^{(v)} \ldots d x_{n-1}^{(v)} \\
\geqslant C_{2} \int_{S_{v}}\left(\sum_{1}^{m} \beta_{j} \psi_{j}^{(v)}\right)^{2} d s, \quad \beta_{j} \in R^{1} .
\end{gathered}
$$

The remaining inequalities of A5(iv) follow from a similar reasoning.

Having verified that the assumptions of Theorem 3.1 are satisfied, let us evaluate the error of the approximation in terms of the parameter $h$ of $\mathrm{Al}(\mathrm{i})$ and $\mathrm{B} 1(\mathrm{i})$. We need the following approximation results:

Proposition 4.1. Let $M_{1}^{h}$ and $M_{2}^{h}$ be defined as above, with $k$ denoting the degree of polynomials in $M_{1}^{h}$, and let $u \in H^{k+1}(\Omega)$ and $\psi \in H^{k+1 / 2}(\partial \Omega)$. Then, if $k>(n-1) / 2$, the following estimates hold

$$
\min _{v \in M_{1}^{h}}\left\{|u-v|_{1, \Omega}+\|u-v\|_{h,-1 / 2, \partial \Omega}\right\} \leqslant C h^{k}\|u\|_{H^{k+1}(\Omega)},
$$

and

$$
\min _{\varphi \in M_{2}^{h}}\left\{\|\psi-\varphi\|_{h, 1 / 2, \partial \Omega}\right\} \leqslant C h^{k}\|\psi\|_{H^{k-1 / 2}(\partial \Omega)} .
$$

Proof. Denote by $N^{h}$ the finite element space associated to the partitioning of $\Omega_{h}$ such that $M_{1}^{h}=\left.N^{h}\right|_{\Omega}$. Further, let $u^{e}$ denote the extension of $u$ to $\Omega_{h}$ such that

$$
\left\|u^{e}\right\|_{H^{k+1}\left(\Omega_{h}\right)} \leqslant C\|u\|_{H^{k+1}(\Omega)} \text {. }
$$

Such an extension exists since $\partial \Omega$ is smooth.

Let $I_{h} u^{e} \in N^{h}$ denote the $k$ th-order Lagrange interpolant of $u^{e}$ on $\Omega_{h}$. If $k>$ $(n-1) / 2, I_{h}$ is a continuous linear operator on $H^{k+1}\left(\Omega_{h}\right)$, and, by the classical results of approximation theory (cf. [5, p. 121]),

$$
\left\|u^{e}-I_{h} u^{e}\right\|_{H^{1}\left(\Omega_{h}\right)} \leqslant C h^{k}\left|u^{e}\right|_{k+1, \Omega_{h}} .
$$

To estimate $\left\|u^{e}-I_{h} u^{e}\right\|_{h,-1 / 2, \partial \Omega}$, let $t_{v} \in \pi^{h}$ be given such that $t_{v} \cap \partial \Omega \neq \varnothing$, and let $J_{v}$ denote the scaling mapping

$$
J_{v}: x \rightarrow \hat{x}=\frac{x}{d\left(t_{v}\right)} .
$$

Further, let $J_{v}\left(t_{v}\right)=\hat{t}_{v}, J_{v}\left(t_{v} \cap \partial \Omega\right)=\hat{\Gamma}_{v}, \hat{u}^{e}(\hat{x})=u^{e}(x)$, and $\hat{I}_{h} \hat{u}^{e}(\hat{x})=I_{h} u^{e}(x)$, $x \in t_{v}$. 
By the assumption B1(ii), if $h$ is small enough, the estimate

$$
\int_{\hat{\Gamma}_{v}}\left(\hat{u}^{e}-\hat{I}_{h} \hat{u}^{e}\right)^{2} d \hat{s} \leqslant C\left\|\hat{u}^{e}-\hat{I}_{h} \hat{u}^{e}\right\|_{H^{1}\left(\hat{t}_{v}\right)}^{2}
$$

holds uniformly for all $\hat{t}_{v}=J_{v}\left(t_{v}\right), t_{v} \in \pi^{h}, t_{v} \cap \partial \Omega \neq \varnothing$. By the approximation properties of $\hat{I}_{h}$ we then have [5]

$$
\int_{\hat{\Gamma}_{v}}\left(\hat{u}^{e}-\hat{I}_{h} \hat{u}^{e}\right)^{2} d \hat{s} \leqslant C\left|\hat{u}^{e}\right|_{k+1, \hat{t}_{v}}^{2}
$$

Upon scaling this back to the original size we get

$$
d\left(t_{v}\right)^{-1} \int_{t_{v} \cap \partial \Omega}\left(u^{e}-I_{h} u^{e}\right)^{2} d s \leqslant C d\left(t_{v}\right)^{2 k}\left|u^{e}\right|_{k+1, t_{v}}^{2}
$$

Summing over $v$ and using B1(i) and B1(iii), (4.11) gives

$$
\left\|u^{e}-I_{h} u^{e}\right\|_{h,-1 / 2, \partial \Omega} \leqslant C h^{k}\left\|u^{e}\right\|_{H^{k+1}\left(\Omega_{h}\right)} .
$$

In view of (4.9), (4.10), and (4.12), the estimate (4.7) follows by choosing $v=\left.I_{h} u^{e}\right|_{\Omega}$.

To prove (4.8), let $\psi \in H^{k}(\partial \Omega)$ and consider a given partitioning $\tau^{h}(\partial \Omega)$. For each $S_{v} \in \tau^{h}(\partial \Omega)$, let $\left\{x_{1}^{(v)}, \ldots, x_{n}^{(v)}\right\}$ be as in (4.3), and assume that $h$ is small enough so that (2.1) through (2.4) hold. For each $S_{v} \in \tau^{h}(\partial \Omega)$, let $D_{v} \subset R^{n-1}$ be a sphere such that $D_{v} \supset U_{v}$, where $U_{v}$ refers to (2.1), and

$$
d\left(D_{v}\right) \leqslant C d\left(S_{v}\right)
$$

for some constant $C$. Further, let

$$
\Gamma_{v}=\left\{\left(x_{1}^{(v)}, \ldots, x_{n}^{(v)}\right) \in \partial \Omega ;\left(x_{1}^{(v)}, \ldots, x_{n-1}^{(v)}\right) \in D_{v}\right\}
$$

and let $\psi_{v}$ be defined on $D_{v}$ as

$$
\psi_{v}\left(x_{1}^{(v)}, \ldots, x_{n-1}^{(v)}\right)=\psi\left(x_{1}^{(v)}, \ldots, x_{n}^{(v)}\right), \quad\left(x_{1}^{(v)}, \ldots, x_{n}^{(v)}\right) \in \Gamma_{v} .
$$

In view of (4.13), there exists for each $v$ a polynomial $\varphi_{\nu}$ on $D_{v}$ of degree $k-1$ such that

$$
\left\|\psi_{v}-\varphi_{v}\right\|_{L_{2}\left(D_{v}\right)} \leqslant C d\left(S_{v}\right)^{k}\left|\psi_{v}\right|_{k, D_{v}},
$$

where $C$ is independent of $v$ (cf. [5, p. 115]). On the other hand, by the assumption Al(ii), the constant $C$ in (4.13) can be chosen so that, for any given $\Gamma \in\left\{\Gamma_{v}\right\}, \Gamma$ intersects $\Gamma_{v}$ for at most $L$ different $\Gamma_{v} \in\left\{\Gamma_{v}\right\}$. Therefore, if $h$ is small enough, (4.14), A1(i), and (2.3) imply

$$
\sum_{v} d\left(S_{v}\right)\left\|\psi_{v}-\varphi_{\nu}\right\|_{L_{2}\left(D_{v}\right)}^{2} \leqslant C h^{2 k+1} \sum_{v}\left|\psi_{v}\right|_{k, D_{v}}^{2} \leqslant C_{1} h^{2 k+1}\|\psi\|_{H^{k}(\partial \Omega)}^{2}
$$

In view of this we have

$$
\min _{\varphi \in M_{2}^{h}}\|\psi-\varphi\|_{h, 1 / 2, \partial \Omega} \leqslant C h^{k+1 / 2}\|\psi\|_{H^{k}(\partial \Omega)}
$$


The estimate (4.8) now follows from interpolation between (4.15) and the trivial estimate

$$
\min _{\varphi \in M_{2}^{h}}\|\psi-\varphi\|_{h, 1 / 2, \partial \Omega} \leqslant h^{1 / 2}\|\psi\|_{L_{2}(\partial \Omega)}
$$

This completes the proof.

Using Proposition 4.1 one can now easily estimate the convergence rate of the Lagrange multiplier method.

THEOREM 4.1. Let $u$ be the solution of problem (1.1) and let $\left(u_{h}, \psi_{h}\right)$ be the solution of (1.3), with $M_{1}^{h}$ and $M_{2}^{h}$ defined as above, and so that $M_{1}^{h}$ contains the polynomials of degree $k$. Then if $f \in H^{k-1}(\Omega)$ and $g \in H^{k+1 / 2}(\partial \Omega)$ in (1.1), and if $k>$ $(n-1) / 2$, the following error bounds hold:

$$
\left\|\left(u, \frac{\partial u}{\partial n}\right)-\left(u_{h}, \psi_{h}\right)\right\|_{X_{\tau h}} \leqslant C h^{k}\left(\|f\|_{H^{k-1}(\Omega)}+\|g\|_{H^{k+1 / 2}(\partial \Omega)}\right),
$$

and

$$
\left\|u-u_{h}\right\|_{L_{2}(\Omega)} \leqslant C h^{k+1}\left(\|f\|_{H^{k-1}(\Omega)}+\|g\|_{H^{k+1 / 2}(\partial \Omega)}\right) .
$$

Proof. The first inequality follows from Theorem 3.1, Proposition (4.1), and the estimate (cf. [7])

$$
\|u\|_{H^{k+1}(\Omega)}+\left\|\frac{\partial u}{\partial n}\right\|_{H^{k-1 / 2}(\partial \Omega)} \leqslant C\left(\|f\|_{H^{k-1}(\Omega)}+\|g\|_{H^{k+1 / 2}(\partial \Omega)}\right), \quad k \geqslant 1 .
$$

The second estimate follows from the ordinary duality argument, see [1].

Institute of Mathematics

Helsinki University of Technology

SF-02150 Espoo 15, Finland

1. I. BABUŠKA, "The finite element method with Lagrange multipliers," Numer. Math., v. 20, 1973, pp. 179-192.

2. I. BABUŠKA \& A. K. AZIZ, "Survey lectures on the mathematical foundations of the finite element method," in The Mathematical Foundations of the Finite Element Method with Applications to Partial Differential Equations (A. K. Aziz, Ed.), Academic Press, New York, 1972.

3. I. BABUŠKA, R. B. KELLOGG \& J. PITKäRANTA, "Direct and inverse error estimates for finite elements with mesh refinements," Numer. Math., v. 33, 1979, pp. 447-471.

4. F. BREZZI, "On the existence, uniqueness and approximations of saddle-point problems arising from Lagrange multipliers," RAIRO, Sér. Anal. Numér., v. 8, 1974, pp. 129-151.

5. P. G. CIARLET, The Finite Element Method for Elliptic Problems, North-Holland, Amsterdam, 1978.

6. J. DOUGLAS, JR., T. DUPONT \& L. WAHLBIN, "The stability in $L^{q}$ of the $L_{2}$-projection into finite element function spaces," Numer. Math., v. 23, 1975, pp. 193-197.

7. J. L. LIONS \& E. MAGENES, Problèmes aux Limites Non Homogènes et Applications, Vol. 1, Dunod, Paris, 1968.

8. J. PITKÄRANTA, "Boundary subspaces for the finite element method with Lagrange multipliers," Numer. Math., v. 33, 1979, pp. 273-289.

9. J. PITKÄRANTA, The Finite Element Method with Lagrange Multipliers for Domains With Corners, Report HTKK-MAT-A141, Institute of Mathematics, Helsinki University of Technology, 1979. 